Instructions for authors, subscriptions and further details:

http://rasp.hipatiapress.com

\title{
El Retraso en la Edad de Jubilación y las Políticas para la Prolongación de la Actividad Laboral Más Allá de la Edad Legal de Jubilación
}

Jordi García-Viña ${ }^{1}$

1) Universidad de Barcelona

Date of publication: July $30^{\text {th }}, 2016$

Edition period: July 2016 - January 2017

To cite this review: García-Viña, J. (2016). El Retraso en la Edad de Jubilación y las Políticas para la Prolongación de la Actividad Laboral Más Allá de la Edad Legal de Jubilación [Review of the Doctoral Thesis].

Research on Ageing and Social Policy, 4(2), 128-129. doi:

10.17583/rasp.2016.2179

To link this review: http://doi.org/10.17583/rasp.2016.2179

PLEASE SCROLL DOWN FOR ARTICLE

The terms and conditions of use are related to the Open Journal System and to Creative Commons Attribution License (CCAL). 
RASP - Research on Ageing and Social Policy Vol. 4 No. 2

July 2016 pp. 128-129

\section{Review}

Barceló Fernández, Jesús (2015). El Retraso en la Edad de Jubilación y las Políticas para la Prolongación de la Actividad Laboral Más Allá de la Edad Legal de Jubilación. Tesis Doctoral. Departamento de Derecho Mercantil, Derecho del Trabajo y de la Seguridad Social. Universidad de Barcelona.

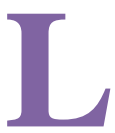

a Tesis Doctoral presenta un estudio del problema de la sostenibilidad de las pensiones públicas de jubilación, no sólo desde el punto de vista de su financiación sino, sobre todo, desde el punto de vista de los pensionistas, los cuales quieren conservar sus derechos de jubilación, y los actuales trabajadores que tienen una legítima expectativa de derecho por la que esperan que, cuando lleguen a la jubilación, puedan contar con unas pensiones adecuadas y suficientes.

Es por ello que, en este trabajo, se analiza en qué medida podría utilizarse el incremento de la edad de jubilación y la prolongación voluntaria de la vida laboral tras cumplir la edad de jubilación, al objeto de conseguir mantener unas pensiones públicas de jubilación sostenibles financieramente a la vez que se garantiza el bienestar de los actuales y futuros pensionistas.

La estructura de la Tesis Doctoral consta de cinco capítulos, siendo el primero relativo a las cuestiones generales, el segundo al estudio de la jubilación en cifras, el tercero aborda la materia relativa a las políticas públicas sobre el retraso en la edad de jubilación, el cuarto es específico sobre la edad legal de jubilación, y el quinto y último sirve como conclusión para aportar toda una serie de medidas legislativas para la promoción del trabajo más allá de la edad ordinaria de jubilación. 
El autor estudia el fenómeno del envejecimiento y sus posibles consecuencias en la sostenibilidad de las pensiones públicas de jubilación, analizando las distintas políticas que se han adoptado tanto en la Unión Europea como en España al objeto de conocer sus efectos y poder hacer propuestas de mejora o de lege ferenda.

El Dr. Barceló Fernández asegura que aplicar como principal medida de Seguridad Social el incremento de la edad ordinaria de jubilación en relación a la esperanza de vida, aunque no se apliquen medidas que supongan la reducción de la pensión media, va a mantener constante el coste de las pensiones en relación al aumento de esperanza de vida, va a asegurar unas pensiones suficientes, adecuadas y dignas para los trabajadores, para cuando alcancen la edad de jubilación, las empresas van a mantener niveles óptimos de productividad, y el consumo interno no se va a ver afectado negativamente, lo que a su vez va a ser positivo para el empleo en general, asegurando la sostenibilidad de las pensiones públicas de jubilación y garantizando el bienestar de los actuales y futuros pensionistas.

En esencia, se trata de una Tesis Doctoral excepcional por las siguientes tres razones:

En primer lugar, por el interés del tema tratado, absolutamente estratégico para, como mínimo, todos los países de la Unión Europea, donde sus niveles de envejecimiento de la población y de problemas en cuanto a la sostenibilidad de las pensiones son comunes.

En segundo lugar, por el análisis realizado, ya que analiza de manera exhaustiva todos los temas y desde diversos puntos de vista.

En tercer lugar, porque es una materia que nos va a acompañar durante los próximos años, ya que forma parte de la esencia de nuestro Estado Social del Bienestar que lo hemos elevado a la categoría de elemento nuclear de la democracia en España.

En conclusión, es necesario felicitar al Dr. Jesús Barceló Fernández por su extraordinario resultado y animarlo a que continúe con su actividad docente e investigadora en la Universidad de Barcelona.

Jordi García-Viña, Catedrático de Derecho del Trabajo y de la Seguridad Social, Universidad de Barcelona. jordi.garcia@ub.edu 